\title{
Aufstockung des Soforthilfeprogramms für Bibliotheken in ländlichen Räumen
}

https://doi.org/10.1515/bd-2020-0090

Das Soforthilfeprogramm für Bibliotheken „Vor Ort für Alle“ erhält weitere Mittel. Initiiert von der Beauftragten der Bundesregierung für Kultur und Medien (BKM), ist das Programm am 15. Mai sehr erfolgreich gestartet und stieß auf eine riesige Nachfrage. Bis Mitte Juli konnten insgesamt bereits 137 Bibliotheken in kleineren Kommunen und Gemeinden Fördermittel erhalten, die ihre Bibliotheken zu „Dritten Orten” weiterentwickeln werden.

Aufgrund des großen Bedarfs hat sich der dbv für eine Aufstockung der Mittel stark gemacht - mit Erfolg. Die insgesamt 1,5 Millionen Euro wurden von der BKM um weitere 700.000 Euro erhöht. Damit können nun zahlreiche weitere Bibliotheken gefördert werden, die bereits einen Antrag gestellt hatten oder sich zeitnah auf der Warteliste des Programms eingetragen haben.

Dazu Prof. Dr. Andreas Degkwitz, Bundesvorsitzender des Deutschen Bibliotheksverbandes e.V. (dbv): „Der große Andrang auf das Förderprogramm ,Vor Ort für Alle' zeigt, wie dringend Bibliotheken in kleinen Kommunen und Gemeinden Investitionen zur Weiterentwicklung ihrer Angebote benötigen. Gerade dort sind Bibliotheken regionale Ankerpunkte für zahlreiche Kultur- und Bildungsangebote. Wir freuen uns daher sehr, dass die Bundesbeauftragte für Kultur und Medien es ermöglicht hat, das Förderprogramm aufzustocken, so dass auch die Bibliotheken, die in der ersten Runde nicht berücksichtig werden konnten, nun ihre Maßnahmen umsetzen können.“

Kulturstaatsministerin Monika Grütters erklärt: „Gerade jetzt, in Zeiten der Corona-Krise, merken wir, wie wichtig Bibliotheken sind, denn sie bieten Zugang zu unserem kulturellen Erbe und zu verlässlichen Informationen. Zugleich sind Bibliotheken auch Begegnungsorte, sie fördern damit den gesellschaftlichen Zusammenhalt. Aus diesem Grund haben wir ein Soforthilfeprogramm für ländliche Räume gestartet, wo Bibliotheken ein existentiell wichtiger Teil der kulturellen Infrastruktur sind. Unser Ziel ist es, Bibliotheken zukunftsfähig zu machen: als Orte der Kultur, des Wissens und der Begegnung.“

Mit dem Programm „Vor Ort für Alle“ werden Vorhaben gefördert, die Bibliotheken in ländlichen Regionen zu zeitgemäßen und lebendigen Kultur- und Begegnungsorten weiterentwickeln. Neben der Bereitstellung von Technik und digitalen Services für Nutzer*innen geht es bei den geförderten Projekten hauptsächlich um die Erhöhung der Aufenthaltsqualität oder die Einrichtung multifunktionaler Bereiche wie z. B. Maker-Spaces. 


\section{Vor Ort für Alle}

Das Soforthilfeprogramm „Vor Ort für alle“ wird aufgrund eines Beschlusses des Deutschen Bundestages im Rahmen des Förderprogramms „Kultur in ländlichen Räumen“ von der Beauftragten der Bundesregierung für Kultur und Medien gefördert. Die Mittel stammen aus dem Bundesprogramm „Ländliche Entwicklung“ (BULE) des Bundesministeriums für Ernährung und Landwirtschaft. Nähere Informationen $\mathrm{zu}$ dem Soforthilfeprogramm finden Sie unter: www. bibliotheksverband.de/dbv/projekte/vor-ort-fuer-alle.html.

\section{Der Deutsche Bibliotheksverband e.V. (dbv)}

Der Deutsche Bibliotheksverband e.V. (dbv) vertritt mit seinen mehr als 2.100 Mitgliedern bundesweit rund 10.000 Bibliotheken mit 25.000 Beschäftigten und 11 Mio. Nutzer^innen. Sein zentrales Anliegen ist es, Bibliotheken zu stärken, damit sie allen Bürger^innen freien Zugang zu Informationen ermöglichen. Der Verband setzt sich ein für die Entwicklung innovativer Bibliotheksleistungen für Wissenschaft und Gesellschaft. Als politische Interessensvertretung unterstützt der dbv die Bibliotheken insbesondere auf den Feldern Informationskompetenz und Medienbildung, Leseförderung und bei der Ermöglichung kultureller und gesellschaftlicher Teilhabe für alle Bürger*innen.

www.bibliotheksverband.de

\section{Pressekontakt}

Kristin Bäßler, Deutscher Bibliotheksverband

Leitung Kommunikation / Pressesprecherin

Tel.: +49(0)30644989925

E-Mail: baessler@bibliotheksverband.de

\section{Projekt „Vor Ort für Alle“}

Mirko Winkelmann, Programmleiter „Vor Ort für alle“

Tel.: +49 (0)30644989919

E-Mail: winkelmann@bibliotheksverband.de, vorortfueralle@bibliotheksver band.de 